\title{
Spectroscopic Determination of Trace Contaminants in High Purity Oxygen
}

\author{
Steven D. Hornung ${ }^{1}$ \\ NASA White Sands Test Facility, Las Cruces, New Mexico, 88004
}

\begin{abstract}
Oxygen used for extravehicular activities (EVA) must be free of contaminants because a difference in a few tenths of a percent of argon or nitrogen content can mean significant reduction in available EVA time. These inert gases build up in the extravehicular mobility unit because they are not metabolized or scrubbed from the atmosphere. Measurement of oxygen purity above $\mathbf{9 9 . 5 \%}$ is problematic, and currently only complex instruments such as gas chromatographs or mass spectrometers are used for these determinations. Because liquid oxygen boil-off from the space shuttle will no longer be available to supply oxygen for EVA use, other concepts are being developed to produce and validate high purity oxygen from cabin air aboard the International Space Station. A prototype optical emission technique capable of detecting argon and nitrogen below $0.1 \%$ in oxygen was developed at White Sands Test Facility. This instrument uses a glow discharge in reduced pressure gas to produce atomic emission from the species present. Because the atomic emission lines from oxygen, nitrogen, and argon are discrete and in many cases well-separated, trace amounts of argon and nitrogen can be detected in the ultraviolet and visible spectrum. This is a straightforward, direct measurement of the target contaminants and may lend itself to a device capable of on-orbit verification of oxygen purity. System design and optimized measurement parameters are presented.
\end{abstract}

\section{Nomenclature}

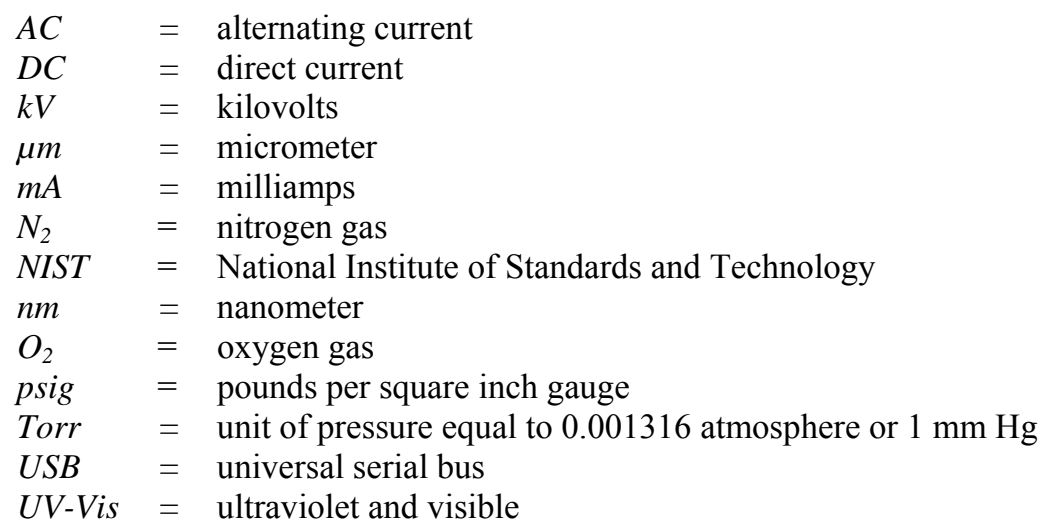

${ }^{1}$ Materials Scientist, Chemistry and Metallurgy Labs, NASA Laboratories Department 201LD, NASA White Sands Test Facility, Las Cruces, New Mexico 88004. AIAA. 


\section{Introduction}

$\mathrm{O}$ xygen used for extravehicular activities (EVA) must be free of contaminants because a difference in a few tenths of a percent of argon or nitrogen content can mean significant reduction in available EVA time. These inert gases can build up in the extravehicular mobility unit (EMU) because they are not metabolized or scrubbed from the atmosphere. Measurement of oxygen purity above $99.5 \%$ is problematic and currently only complex instruments such as gas chromatographs or mass spectrometers are used for these determinations. Because liquid oxygen boil-off from the space shuttle will no longer be available to supply oxygen for EVA use, other concepts are being developed to produce high purity oxygen from cabin air aboard the International Space Station.

White Sands Test Facility (WSTF) has developed a prototype optical emission technique capable of detecting argon and nitrogen below $0.1 \%$ in oxygen. This instrument uses a glow discharge in reduced pressure gas to produce atomic emission from the species present. Because the atomic emission lines from oxygen, nitrogen, and argon are discrete and in many cases well-separated, trace amounts of argon and nitrogen can be detected in the ultraviolet and visible spectrum. Spectra of trace argon in air (naturally occurring at $0.9 \%$ ) were observed as early as the late 1800 s using the electronics and optical instruments of the era.

A glow discharge is plasma formed in a low pressure (1 to 10 Torr) gas cell between two electrodes. Depending on the configuration, voltages ranging from 200 volts and above are required to sustain the discharge. In the discharge region, the gas is ionized and a certain population is in the excited state. Light is produced by the transitions from the excited states formed in the plasma to the ground state. The spectrum consists of discrete, narrow emission lines for the atomic species and broader peaks that may appear as a manifold for molecular species such as $\mathrm{O}_{2}$ and $\mathrm{N}_{2}$, the wavelengths and intensities of which are a characteristic of each atom. Glow discharge spectra of oxygen, nitrogen, and argon are shown in Figure 1. The oxygen emission is dominated by two peaks at 777 and $844 \mathrm{~nm}$ corresponding to the ${ }^{5} \mathrm{P}->$ ${ }^{5} \mathrm{~S}^{0}$ and ${ }^{3} \mathrm{P}->{ }^{3} \mathrm{~S}^{0}$ transitions.

In a mixture of gases at a fixed pressure, the intensity of lines in the spectrum depends on the concentration of gas, the total pressure of the system, and the discharge current through the plasma.
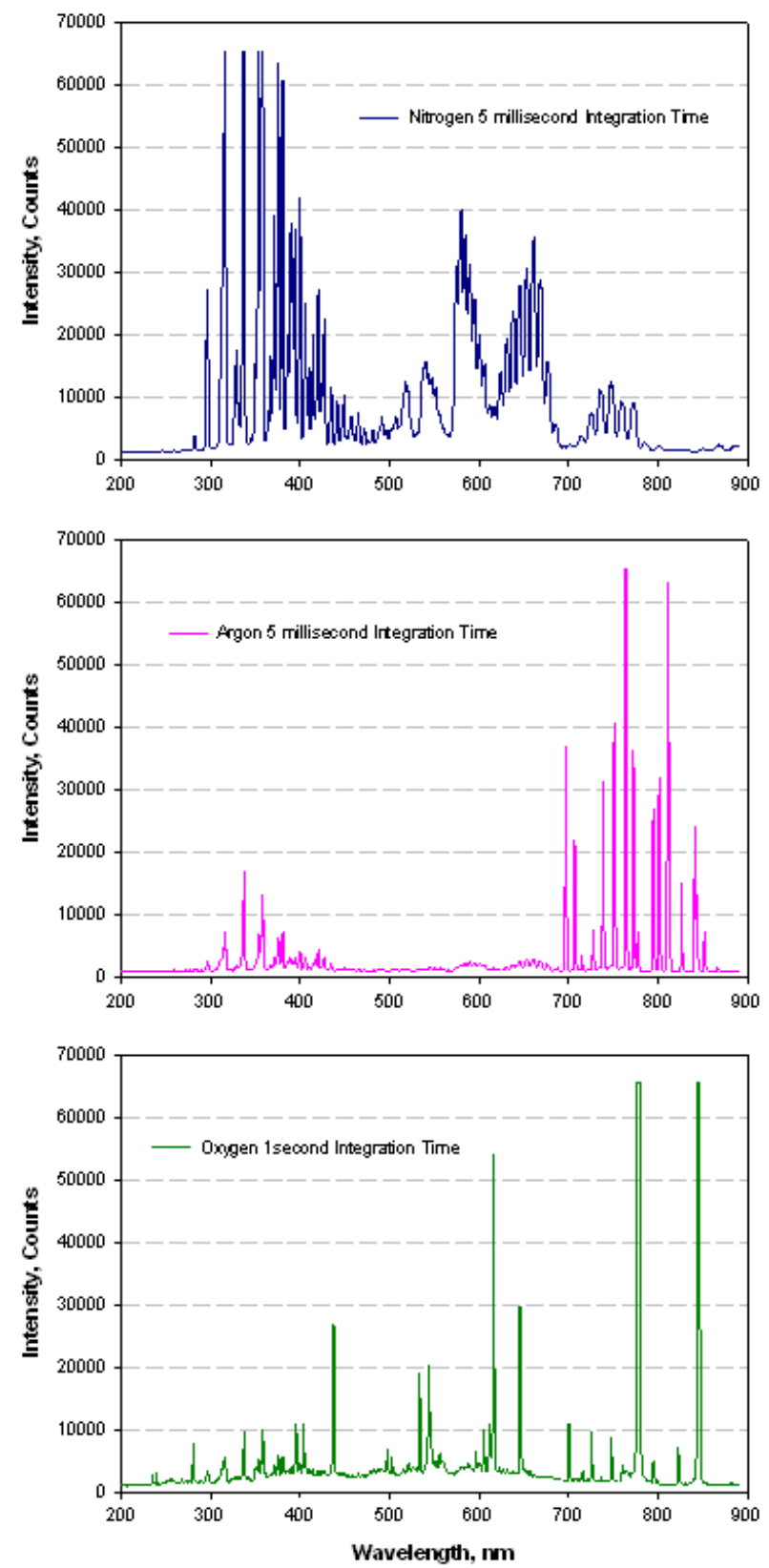

Figure 1. Reference glow discharge emission spectra for pure nitrogen, argon, and oxygen taken at 1.3 Torr. 


\section{Experimental}

A block diagram of the system is shown in Figure 2 and a photograph of the prototype in Figure 3. A quartz capillary tube with stainless steel end fittings forms the glow discharge tube. The sample gas is introduced into the glow discharge cell using an adjustable vacuum leak valve. From the glow discharge cell, the sample gas passes a vacuum gauge, the downstream valve, and then the vacuum pump. During operation, the pressure in the glow discharge cell is maintained between 0.5 and 10 Torr using the adjustable leak valve and the downstream valve. In the proof-of-concept testing, the discharge power was supplied by a $5 \mathrm{kV} \mathrm{AC}, 10 \mathrm{~mA}$ power supply. Light from the discharge is collected by a lens and coupled to a UV-Vis fiber optic cable. This cable directs the light from the glow discharge into the Ocean Optics $\mathrm{Jaz}^{\mathbb{B} *}$ spectrometer. The spectrometer detects in the 200 to $850 \mathrm{~nm}$ region with a spectral resolution of $1.5 \mathrm{~nm}$ using a $25 \mu \mathrm{m}$ entrance slit. The spectrometer is connected to a data acquisition computer via a USB cable. For this work, Ocean Optics SpectraSuite ${ }^{\mathbb{B} *}$ software was used for the data acquisition, setting parameters such as wavelength range, integration times, and scans to average.

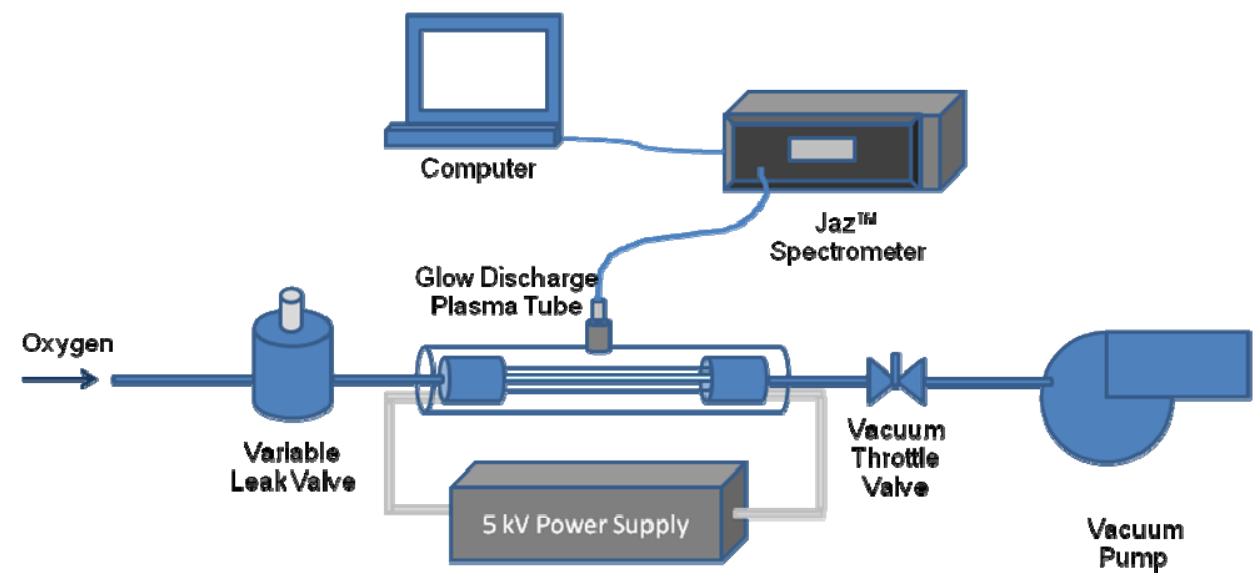

Figure 2. Schematic diagram of the glow discharge system.

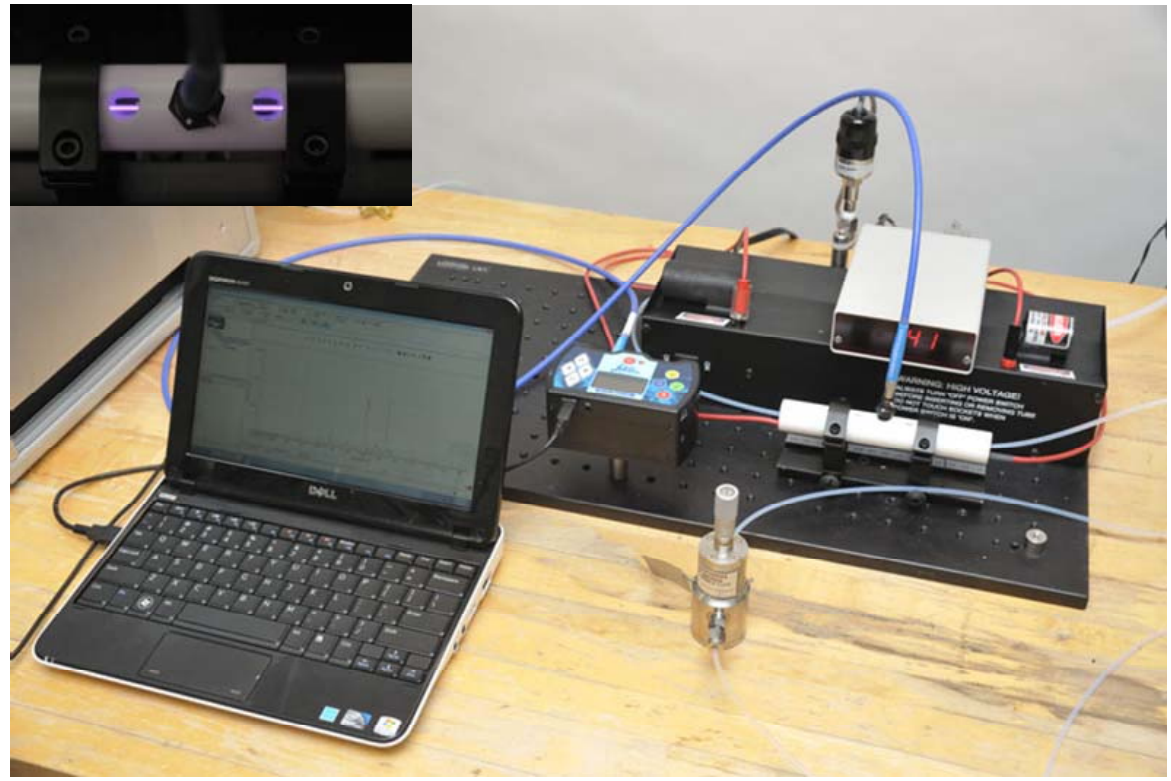

wstf0211e01193 and -01196

Figure 3. Photograph of the prototype system with inset showing glow discharge region. The vacuum pump is not shown.

\footnotetext{
${ }^{*} \mathrm{Jaz}^{\circledR}$ and SpectraSuite ${ }^{\circledR}$ are registered trademarks of Ocean Optics, Inc., Dunedin, Florida.
} 
Gas standards containing trace amounts of argon or nitrogen in $99.999 \%$ pure oxygen were prepared by partial pressure gas dilution. The standard containers were evacuated to less than 0.05 Torr and held at vacuum for a minimum of 30 minutes. Argon or nitrogen was admitted to the gas manifold and the pressure measured using a NIST-traceable calibrated Baratron ${ }^{\mathbb{} \dagger}$ pressure transducer. Gas standards were prepared at $1,0.5,0.1,0.05$, and 0.01 percent by volume of argon in oxygen at a final pressure of 45 psig.

\section{Results and Discussion}

A tube pressure of 1.3 Torr was chosen for initial tests due to the low spectral intensity of the oxygen lines; at pressure below 1 Torr, oxygen emission intensity increases dramatically. Emission spectra were obtained for each standard at 2 seconds spectrometer integration time. This relatively long integration time serves to increase the peak intensity as well as averaging out the noise caused by fluctuations in the emission spectrum. The spectra of these standards were surveyed to select candidate lines to quantitate the amount of argon present. The ideal line would be one of the most intense argon lines and well resolved or separated from the more predominant oxygen lines. Figure 4 shows the spectrum of oxygen and oxygen with $1 \%$ argon. Argon lines are noted at $696,706,738,750,763,801$, and

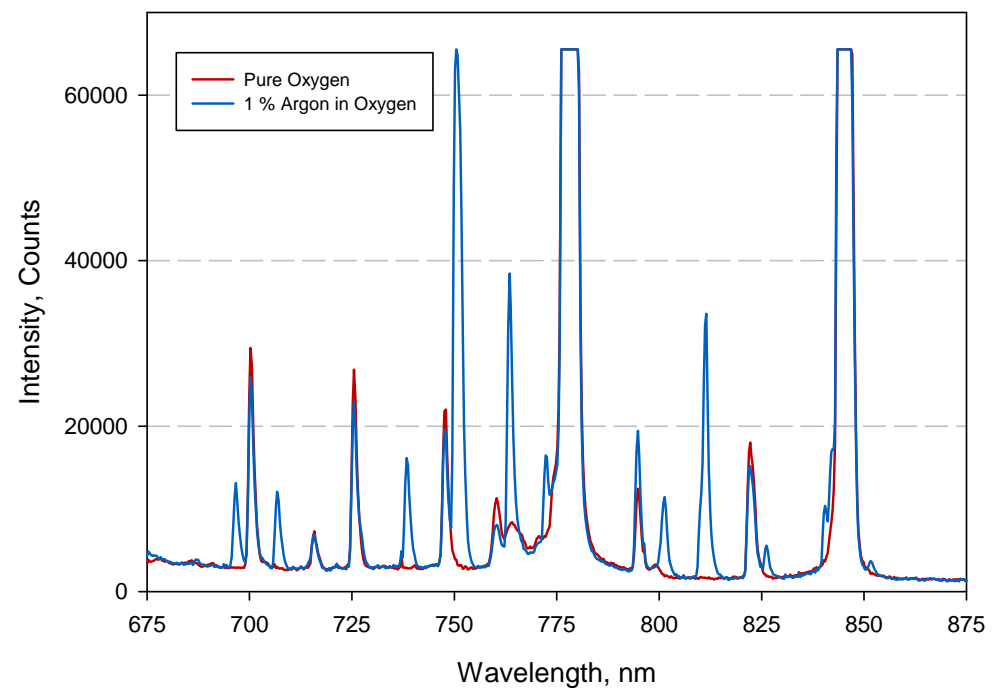

Figure 4. Glow discharge emission spectra of pure oxygen and oxygen with $1 \%$ added argon.

$811 \mathrm{~nm}$. The argon lines best separated

from the oxygen lines are found at 738,801 , and $811 \mathrm{~nm}$. The argon line at $750 \mathrm{~nm}$ has some overlap from an adjacent oxygen peak, but because it is the most intense argon emission line, it is worth considering.

The next step is to determine the approximate detection limit for each peak used. For a peak to be at the detection limit, it must:

- Be recognizable as a peak

- Be resolved from other peaks

- Have a peak intensity three times the standard deviation of background noise in the region of the peak

Figure 5 shows the region around the well-resolved argon line at $811 \mathrm{~nm}$ for $0.1,0.05,0.01$, and pure oxygen. The peak corresponding to $0.01 \%$ argon is just above the baseline of pure oxygen and the signal-to-noise ratio is 2.6, indicating the detection limit is between 0.05 and $0.01 \%$. Table 1 summarizes the results of the detection limit determination for each peak.

\footnotetext{
${ }^{\dagger}$ Baratron ${ }^{\circledR}$ is a registered trademark of MKS Instruments, Inc., Andover, Massachusetts.
} 


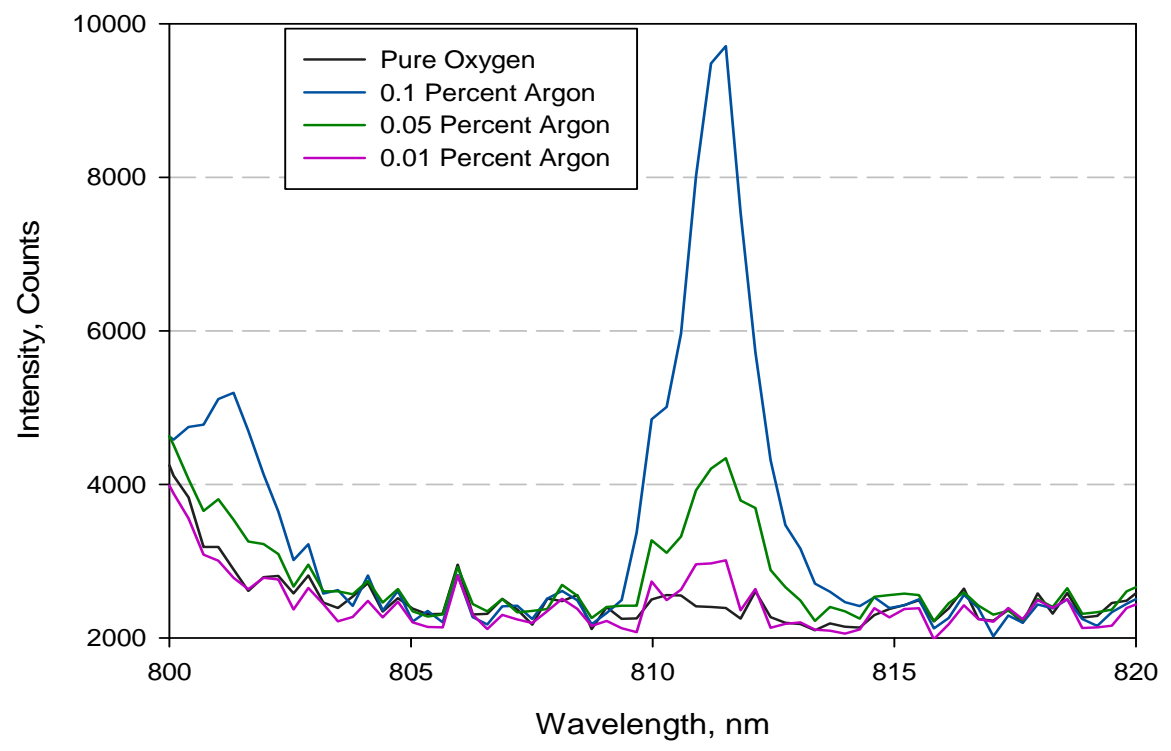

Figure 5. Region around the $811 \mathrm{~nm}$ peak for $0.1,0.05,0.01$, and pure oxygen.

Table 1. Detection Limit, Peak Height, and Signal-to-Noise Ratio for each Peak

\begin{tabular}{cccc}
\hline $\begin{array}{c}\text { Peak, } \\
\text { nm }\end{array}$ & $\begin{array}{c}\text { Detection Limit, } \\
\text { Percent }\end{array}$ & $\begin{array}{c}\text { Peak - Background, } \\
\text { Counts }\end{array}$ & $\begin{array}{c}\text { Signal-to-Noise } \\
\text { Ratio }\end{array}$ \\
\hline 738 & 0.05 & 396 & 2.5 \\
750 & 0.05 & 836 & 4.1 \\
801 & 0.05 & 443 & 2 \\
811 & $0.01 \leq \leq 0.05$ & 349 & 2.6 \\
\hline
\end{tabular}

Spectra were obtained for each standard and the peak emission for each of these lines was tabulated and the adjacent background continuum signal subtracted. To account for fluctuations in the overall spectra due to changes in discharge current, the background corrected signal from each peak was normalized by dividing by the intensity of an oxygen peak in the same region. The oxygen emission peak at $822 \mathrm{~nm}$ was chosen because its intensity is on the same order of magnitude as the argon peaks of interest. These normalized peak heights are plotted as a function of argon concentration in Figure 6. Linear regressions for each peak are plotted on the graph as well. The results of these linear regressions are summarized in Table 2. 


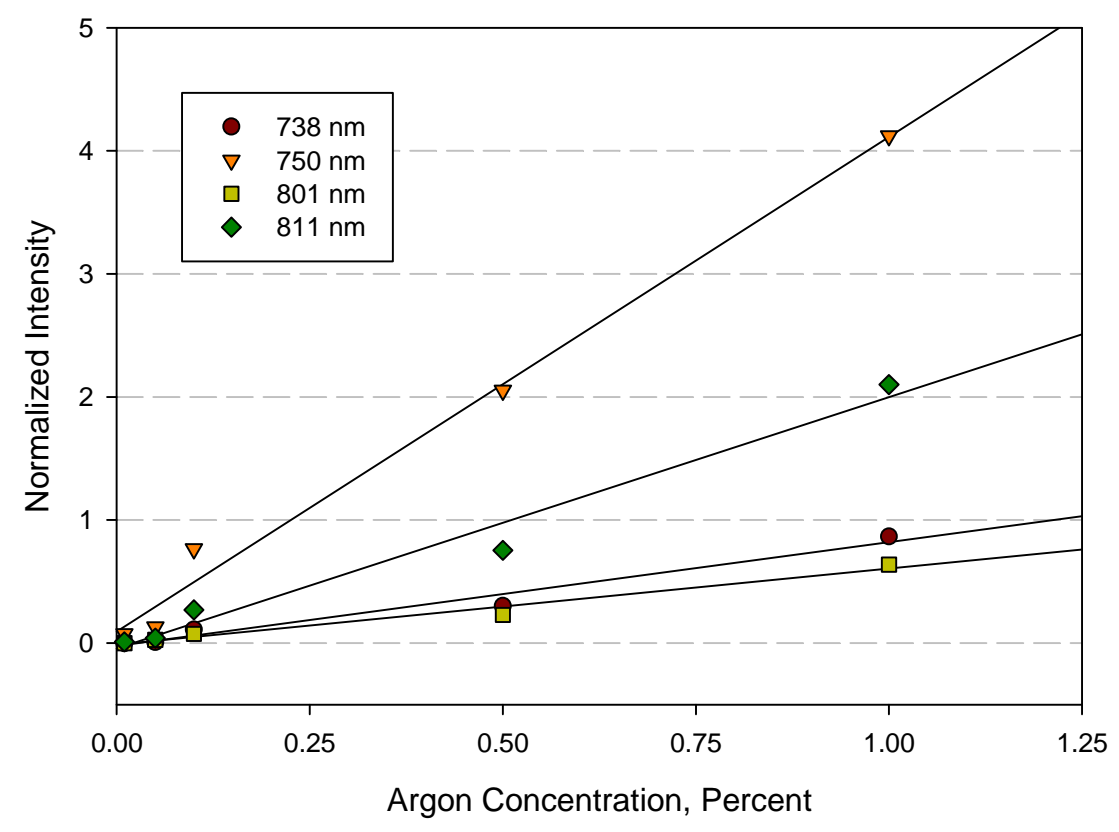

Figure 6. Normalized peak intensities as a function of argon concentration in oxygen plotted for the argon emission peaks at 738, 750, 801 , and $811 \mathrm{~nm}$.

Table 2. Results of linear regression analysis as a function of argon emission peak

\begin{tabular}{cccc}
$\begin{array}{c}\text { Argon Emission Peak } \\
\text { Wavelength, }\end{array}$ & Regression & Regression & $\begin{array}{c}\text { Coefficient of } \\
\text { Determination, } \\
\text { nm }\end{array}$ \\
\hline 738 & Slope & Y Intercept & $\mathrm{r}^{2}$ \\
750 & 0.84 & -0.03 & 0.97 \\
801 & 4.0 & 0.09 & 0.99 \\
811 & 0.62 & -0.01 & 0.98 \\
\hline
\end{tabular}

\section{Summary}

This work represents a proof-of-concept investigation into using a glow discharge emission system to detect and quantitate trace amounts of argon in pure oxygen. A similar analysis will need to be done for nitrogen. Optimization of experimental parameters such as operating pressure, discharge current and voltage, and spectrometer integration time need to be further investigated. A redesigned discharge cell that will use a lower voltage DC power supply with a higher discharge current is being designed to provide a spectrally brighter, lower noise glow discharge. 